\title{
Star Formation Efficiency at Intermediate Redshift
}

\author{
F. Combes ${ }^{1}$, S. García-Burillo ${ }^{2}$, J. Braine ${ }^{3}$, E. Schinnerer ${ }^{4}$, \\ F. Walter ${ }^{4}$, L. Colina ${ }^{5}$ \\ ${ }^{1}$ Observatoire de Paris, LERMA (CNRS:UMR8112), \\ 61 Av. de l'Observatoire, F-75014, Paris, France \\ email: francoise.combes@obspm.fr \\ ${ }^{2}$ Observatorio Astronómico Nacional (OAN)-Observatorio de Madrid, \\ Alfonso XII, 3, 28014-Madrid, Spain \\ ${ }^{3}$ Laboratoire d'Astrophysique de Bordeaux, UMR 5804,Université Bordeaux I, \\ BP 89, 33270 Floirac, France \\ ${ }^{4}$ Max-Planck-Institut für Astronomie (MPIA), Königstuhl 17, 69117 Heidelberg, Germany \\ ${ }^{5}$ Departamento de Astrofisica, Centro de Astrobiologia (CSIC/INTA), \\ Torrejón de Ardoz, 28850 Madrid, Spain
}

\begin{abstract}
Star formation is evolving very fast in the second half of the Universe, and it is as yet unclear whether this is due to evolving gas content, or evolving star formation efficiency (SFE). We have carried out a survey of ultra-luminous galaxies (ULIRG) between $\mathrm{z}=0.2$ and 1 , to check the gas fraction in this domain of redshift which is still poorly known. Our survey with the IRAM-30m detected 33 galaxies out of 69 , and we derive a significant evolution of both the gas fraction and SFE of ULIRGs over the whole period, and in particular a turning point around $\mathrm{z}=0.35$. The result is sensitive to the CO-to- $\mathrm{H}_{2}$ conversion factor adopted, and both gas fraction and SFE have comparable evolution, when we adopt the low starburst conversion factor of $\alpha=0.8 \mathrm{M}_{\odot}\left(\mathrm{K} \mathrm{km} \mathrm{s}^{-1} \mathrm{pc}^{2}\right)^{-1}$. Adopting a higher $\alpha$ will increase the role of the gas fraction. Using $\alpha=0.8$, the SFE and the gas fraction for $\mathrm{z} \sim 0.2-1.0$ ULIRGs are found to be significantly higher, by a factor 3, than for local ULIRGs, and are comparable to high redshift ones. We compare this evolution to the expected cosmic $\mathrm{H}_{2}$ abundance and the cosmic star formation history.
\end{abstract}

Keywords. galaxies: evolution — galaxies: ISM — galaxies: interactions — galaxies: starburst — radio lines: galaxies

\section{Introduction}

Star formation (SF) was proceeding at a much faster rate in galaxies in the first half of the universe's history, and the most striking feature in the cosmic SF rate density is the decline by a factor $\sim 10$ since $\mathrm{z}=1$ (Madau et al. 1998, Hopkins \& Beacom 2006). Several factors could be invoked to explain such a behavior: first the gas fraction in star forming galaxies is likely to have been higher in the past, as already suggested by CO surveys, tracing the molecular gas content of galaxies. Locally, the gas fraction for giant spirals is about $7-10 \%$ (Leroy et al. 2008, Saintonge et al. 2011a), while at $\mathrm{z} \sim 1.2$ it increases to $34 \pm 5 \%$ and at $\mathrm{z} \sim 2.3$ to $44 \pm 6 \%$ (Tacconi et al. 2010, Daddi et al. 2010). Second, the star formation efficiency might have been higher in the past, due to the dynamical trigger of galaxy interactions, whose frequency increases with redshift (e.g. Conselice et al.2009, Kartaltepe et al. 2010), and also the more violent instabilities in more unstable disks, with lower bulge-to-disk ratios. The star formation efficiency (SFE) defined as the ratio of SFR to gas content, has been observed to increase with redshift (e.g. Greve et al. 2005), even for 
the most extreme starbursts, represented by ultra-luminous infrared galaxies (ULIRG). This tendency is however supported mainly by comparing local and high-z galaxies, at $\mathrm{z}>1$, and very little is known about the molecular gas content of galaxies at intermediate redshift between $\mathrm{z}=0.2$ and 1 . This $\mathrm{CO}$ desert is mainly due to observational difficulties, and motivated our $\mathrm{CO}$ survey of starburst galaxies in this redshift range. A first study at $0.2<\mathrm{z}<0.6$ (Combes et al. 2011) has indeed confirmed a strong increase of SFE in this redshift range.
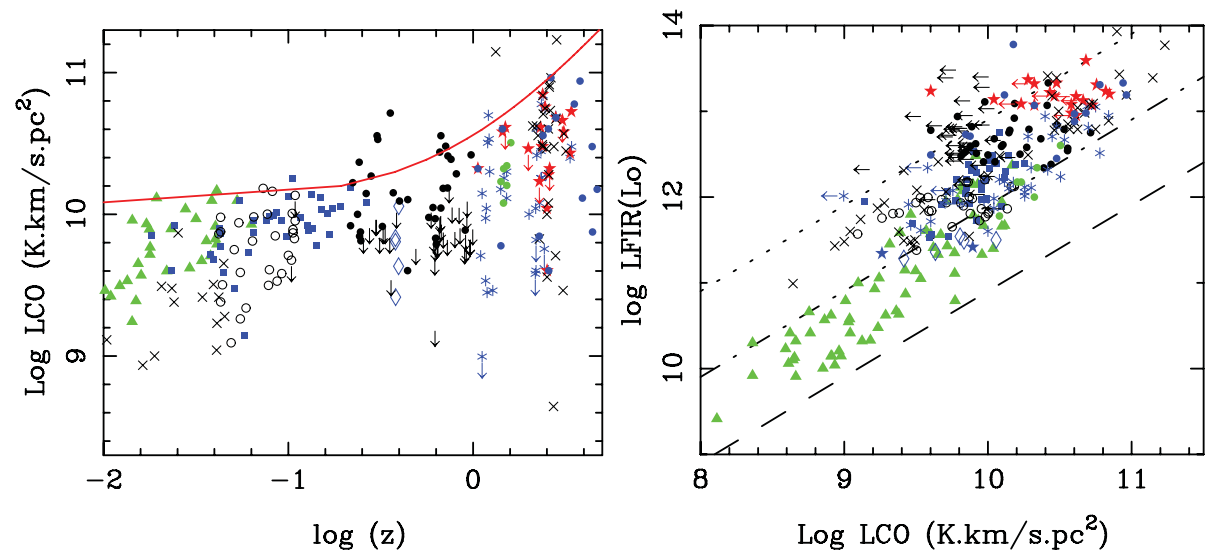

Figure 1. Left: $\mathrm{CO}$ luminosities, a proxy for total $\mathrm{H}_{2}$ masses, versus redshift, for the objects of our sample (filled black circles, and arrows as upper limits), compared to various data from the literature: green triangles are from Gao \& Solomon (2004), blue squares from Solomon et al. (1997), open circles from Chung et al. (2009), blue diamonds from Geach et al. (2009, 2011), black crosses from Iono et al. (2009), red stars, from Greve et al. (2005), green filled circles from Daddi et al.(2010), blue asterisks from Genzel et al.(2010), and blue filled circles from Solomon \& vanden Bout (2005). For illustration purposes only, the red curve is the power law in $(1+\mathrm{z})^{1.6}$ for $\Omega_{\mathrm{H}_{2}} / \Omega_{\mathrm{HI}}$ proposed by Obreschkow \& Rawlings (2009). Right: Correlation between far infrared and $\mathrm{CO}$ luminosities for the same data. The 3 lines are for $\mathrm{L}_{\mathrm{FIR}} / \mathrm{M}\left(\mathrm{H}_{2}\right)=10,100$ and $1000 \mathrm{~L} \odot / \mathrm{M}_{\odot}$ from bottom to top, assuming a conversion factor $\alpha=0.8 \mathrm{M}_{\odot}(\mathrm{K} \mathrm{km} \mathrm{s}$ $\left.\mathrm{pc}^{2}\right)^{-1}$. The three lines correspond to gas depletion time-scales of 580 (bottom), 58 (middle) and 5.8 Myr (top).

\section{Star formation efficiency and gas fraction}

We have now completed our CO survey of 69 starburst galaxies with $0.2<\mathrm{z}<1.0$, and obtained a global detection rate of $48 \%$. Since the conversion factor is a key parameter in this study, we have obtained CO detections in several J-lines for a dozen of galaxies, and found different excitation for the gas. When the gas mass could be derived from the dust emission however, the resulting values supported our adoption of the $\alpha=0.8$ conversion factor (Combes et al.2012). Mapping of some galaxies with the IRAM interferometer showed that the molecular gas is extended at kpc scales, and not only confined to a nuclear component (Combes et al.2006, and in prep.).

Figure 1 shows that the data gap at intermediate redshift is now filled. The CO luminosities of starburst galaxies display an envelope which is constantly rising with redshift in this interval. The FIR to CO correlation is non-linear, and define gas depletion time between 6 and 600 Myr (Fig 1 right).

The gas fraction requires the determination of stellar mass, which was obtained through SED-fitting of optical and near-infrared luminosities (Combes et al. 2012). We define the 

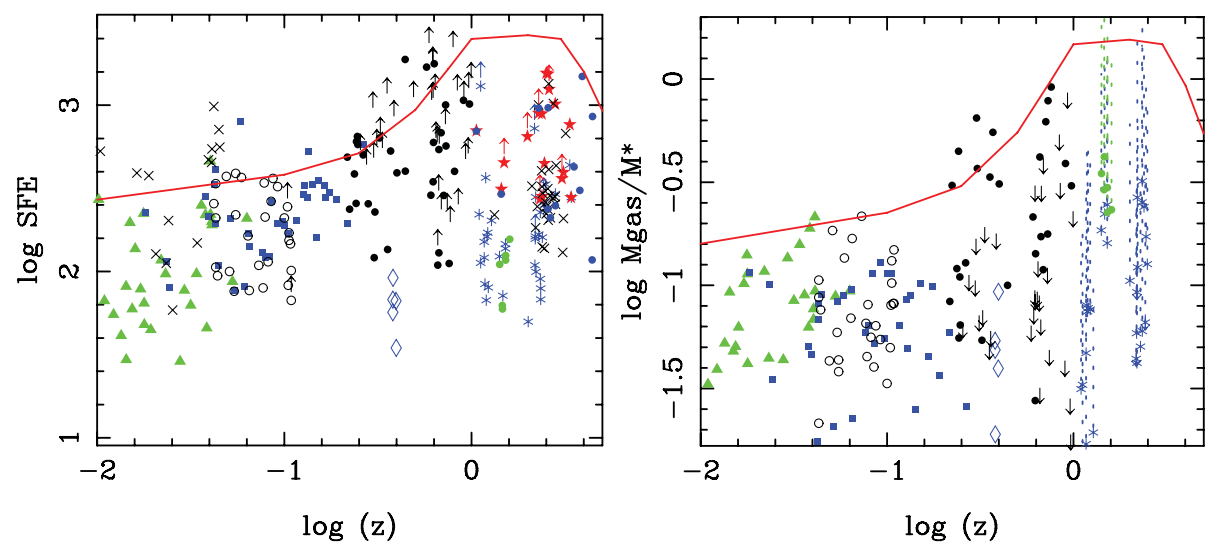

Figure 2. Left: The star formation efficiency versus redshift $\mathrm{z}$. The symbols are the same as in Fig 1. The red curve is a schematic line summarizing the evolution of cosmic star formation density, from the compilation by Hopkins \& Beacom (2006), complemented with recent work by Kistler et al. (2009) and Bouwens et al. (2008). This indicative curve is logarithmic and can be translated vertically. Right: The gas to stellar mass ratio vs redshift, with the same data. The points of the high-z samples (blue asterisks and green dots), have been continued by a dotted line joining the two extreme values of gas fraction, obtained with conversion factors $\alpha=0.8$ and $4.6 \mathrm{M}_{\odot}\left(\mathrm{K} \mathrm{km} \mathrm{s}^{-1} \mathrm{pc}^{2}\right)^{-1}$.

star formation efficiency as the ratio of far infrared luminosity (proxy of star formation rate) and the molecular mass derived from the CO luminosity, with a constant conversion factor $\alpha=0.8 \mathrm{M}_{\odot}\left(\mathrm{K} \mathrm{km} \mathrm{s}^{-1} \mathrm{pc}^{2}\right)^{-1}$. The SFE and gas fraction are plotted versus redshift and compared to available data in Fig 2. Both display an envelope which rises with redshift in the intermediate range between 0.2 and 1.0, the range where the cosmic star formation rate density is remarkably increasing by an order of magnitude. These trends are also visible in the bin-averages of the data, taking or not the upper limits into account (cf Fig 3).
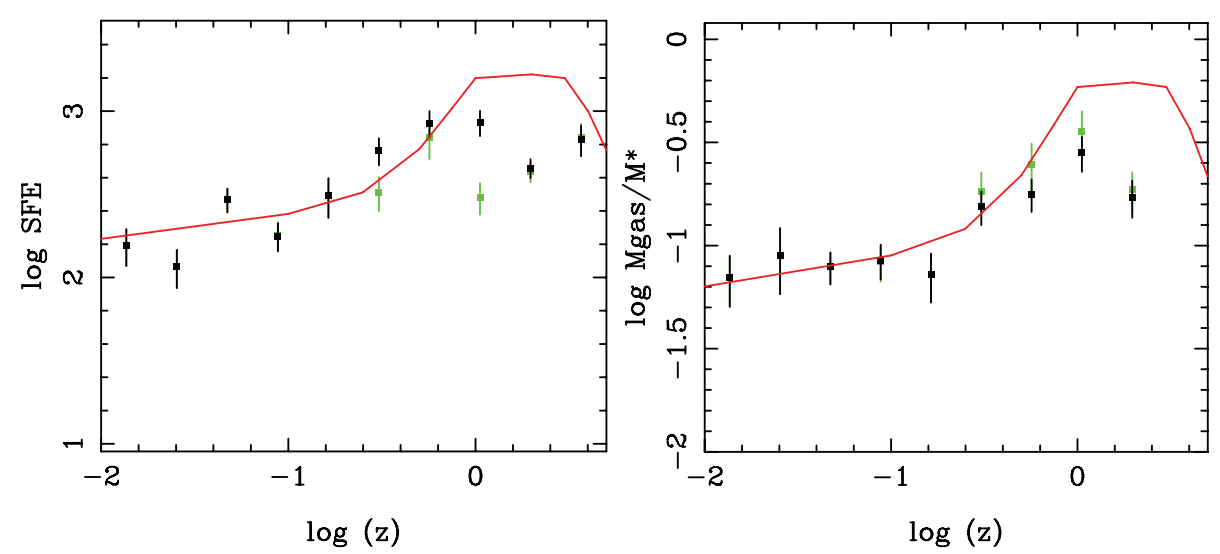

Figure 3. Evolution with redshift of averaged quantities, SFE at left, and gas to stellar mass ratio at right. The average of detected points only is plotted in green, and with the $3 \sigma$ upper limits in black (for high-z samples only). The error bars are based on Poisson noise. The red line is the same as in Figure 2. 


\section{Conclusion}

The increase of both star formation efficiency and gas fraction with redshift in the intermediate range for starburst galaxies suggests that both factors play a role in driving the evolution of the cosmic star formation rate density. The relative influence of each physical quantity is strongly linked to the $\mathrm{CO}-$ to- $\mathrm{H}_{2}$ conversion factor. If a standard Milky Way ratio is used for these galaxies, their gas fraction will then dominate the evolution. We have tried to relate the star formation efficiency to the compactness of the starburst, measured from the half-light radius in the I-band (corresponding to the blue band in the rest frame). There is indeed an anticorrelation of SFE with half-light radius, but with a large scatter. The SFE was also plotted with respect to the specific star formation rate (or SFR per unit stellar mass), and compared to the locus of local "normal" star forming galaxies from the COLD GASS survey (Saintonge et al.2011b). The starburst galaxies are globally located below, meaning that their gas content is significantly higher.

\section{References}

Bouwens R. J., Illingworth G. D., Franx M., \& Ford H. 2008, ApJ 686, 230

Chung, A., Narayanan, G., Yun, M. S., Heyer, M., \& Erickson, N. R. 2009, AJ 138, 858

Combes, F., García-Burillo, S., Braine, J. et al. 2006, A\&\&A 460, L49

Combes, F., García-Burillo, S., Braine, J. et al.2011, Aछ A 528, A124

Conselice, C. J., Yang, C., \& Bluck, A. F. L. 2009, MNRAS 394, 1956

Daddi E., Bournaud, F., Walter, F. et al. 2010 ApJ 713, 686

Gao Y. \& Solomon P. M. 2004 ApJS 152, 63

Geach, J. E., Smail I., Coppin K. et al.2009, MNRAS 395, L62

Geach, J. E., Smail I., Moran S. M. et al. 2011, ApJ 730, L19

Genzel, R., Tacconi, L. J., Gracia-Carpio, J. et al.2010, MNRAS 407, 2091

Greve. T. R., Bertoldi, F., Smail, I. et al. 2005, MNRAS 359, 1165

Hopkins, A. M. \& Beacom J. F. 2006, ApJ, 651, 142

Iono, D., Wilson, C. D., Yun, M. S. et al. 2009, ApJ, 695, 1537

Kartaltepe, J. S., Sanders, D. B., Le Floc'h, E. et al. 2010, ApJ 721, 98

Kistler M. D., Yüksel H., Beacom J. F. et al. 2009, ApJ 705, L104

Leroy, A. K., Walter, F., Brinks, E. et al. 2008, AJ 136, 2782

Madau P., Pozzetti L., \& Dickinson M. E. 1998, ApJ 498, 106

Obreschkow, D., \& Rawlings, S. 2009 ApJ 696, L129

Saintonge, A., Kauffmann, G., Kramer, C. et al.2011a, MNRAS 415, 32

Saintonge, A., Kauffmann, G., Wang J. et al.2011b, MNRAS 415, 61

Solomon P., Downes D., Radford S., \& Barrett J. 1997, ApJ 478, 144

Solomon P. \& Vanden Bout P. A. 2005, ARAA 43, 677

Tacconi L. J., Genzel R., Neri R. et al.2010, Nature 463, 781 